\title{
Rayleigh-Bénard convection in air: out-of-plane vorticity from stereoscopic PIV measurements
}

\author{
V. Valori ${ }^{1 *}$, A. Thieme ${ }^{1}$, C. Cierpka ${ }^{1}$, J. Schumacher ${ }^{1,2}$ \\ ${ }^{1}$ Technische Universität Ilmenau, Institute of Thermodynamics and Fluid Mechanics, D-98684 Ilmenau, \\ Germany \\ ${ }^{2}$ New York University, Tandon School of Engineering, New York City, NY 11201, USA \\ *valentina.valori@tu-ilmenau.de
}

\begin{abstract}
We present results from stereoscopic PIV measurements in a Rayleigh-Bénard convection (RBC) cell filled with (compressed) air at Rayleigh numbers: $R a=1.5 \times 10^{4}, 2 \times 10^{4}, 1 \times 10^{5}, 2 \times 10^{5}, 5 \times 10^{5}$, and Prandtl number $\operatorname{Pr} \simeq 0.7$. The three largest Rayleigh numbers are obtained pressurising the whole set-up including cameras and objective lenses, up to 4.5 bars.

The main goal of this study is to reproduce DNS data that are acquired at the same Rayleigh numbers to study far-tail events of the out-of-plane vorticity component $\left(\omega_{z}\right)$.

The measurements are performed in a RBC cell with aspect ratio $\Gamma=W / H=10$, where $W$ is the width and $H=3 \mathrm{~cm}$ is the height of the domain. The cell is equipped with a transparent bottom plate heated by a thin oxide layer (for details see Kästner et al. (2018)), which allows us to measure 3C2D velocity fields on a horizontal plane at mid height of the cell. The RBC cell set-up is inserted in the SCALEX facility of TU Ilmenau, a pressure vessel with several optical accesses that can be pressurised up to 10 bars.
\end{abstract}

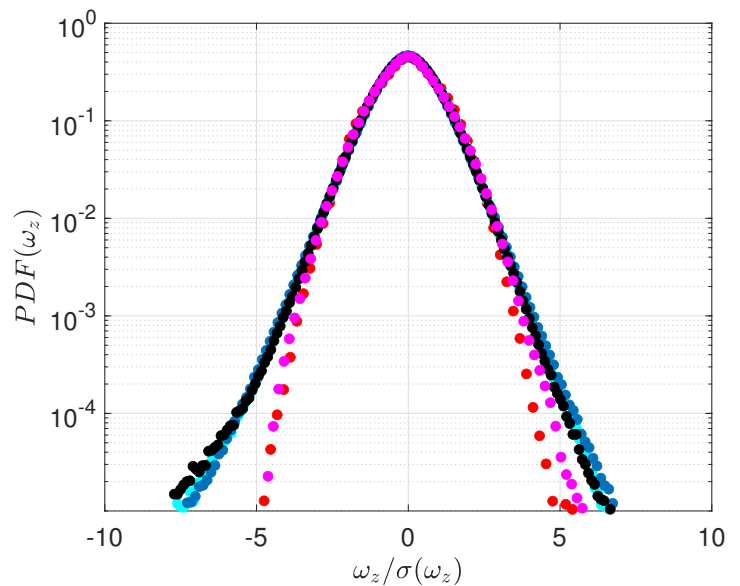

(a) stereo PIV

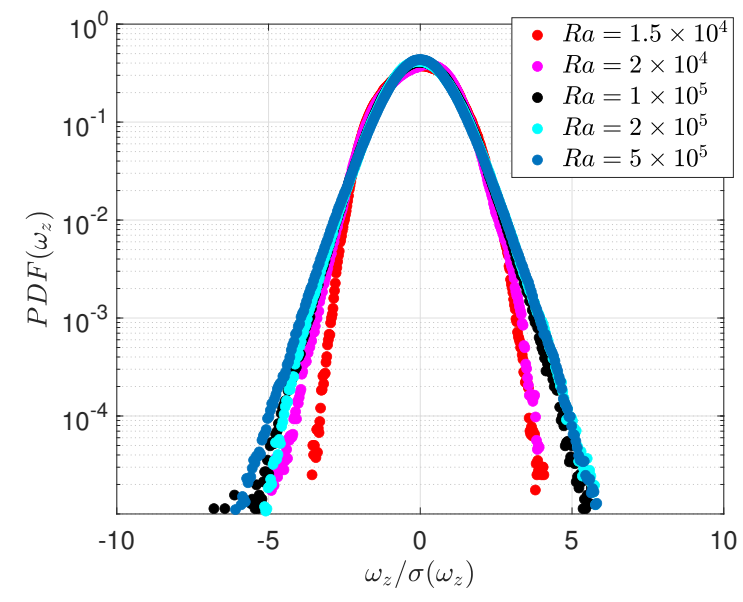

(b) DNS

Figure 1: PDFs of $\omega_{z}$ from stereo PIV and DNS data at $R a=1.5 \times 10^{4}, 2 \times 10^{4}, 1 \times 10^{5}, 2 \times 10^{5}, 5 \times 10^{5}$

The experiments aim firstly at improving the quality of previous measurements performed in the same set-up [Kästner et al. (2018), Cierpka et al. (2019)], regarding the accuracy of the out-of-plane velocity component. This has been realised by positioning the cameras at a larger stereo angle (about $25^{\circ}$ ), which is possible by placing them inside the pressure vessel. Major challenges of the current measurements are caused by optical distortions due to the temperature gradients that are typical for thermal convection (see Valori (2018), Valori et al. (2019)). 


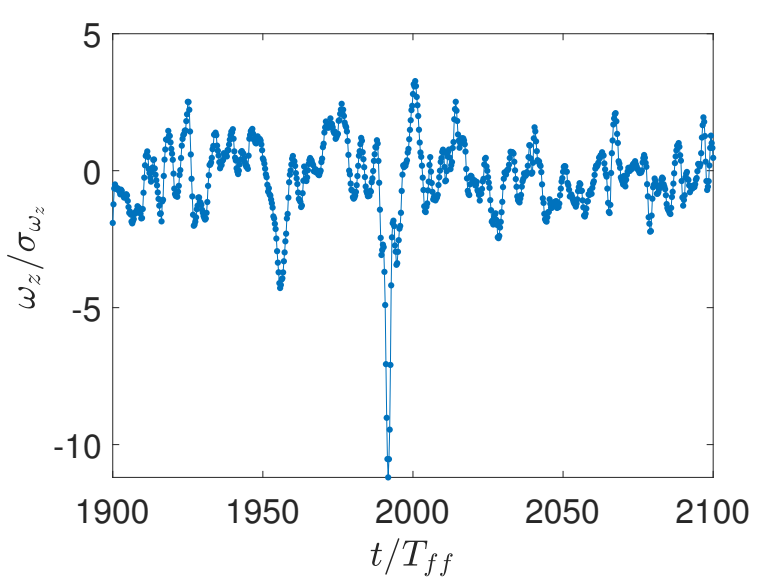

(a) Time evolution of $\omega_{z}$ at $\left(x_{e}, y_{e}\right)$

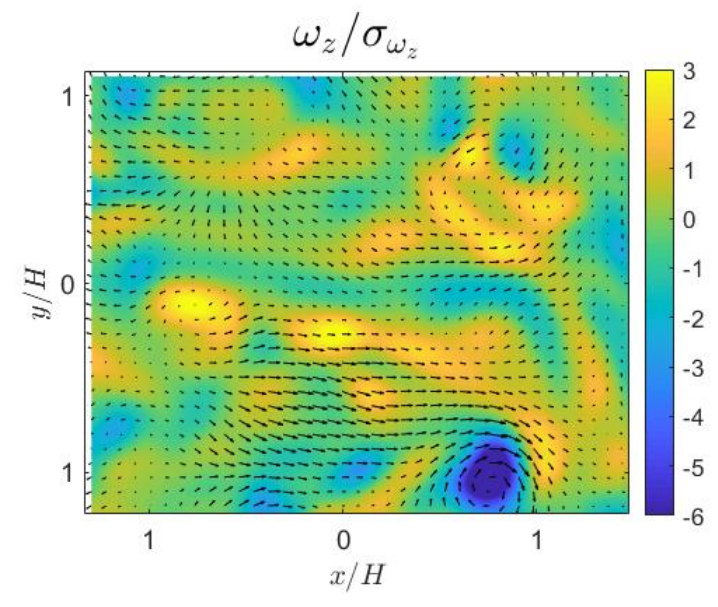

(b) Spatial structutre at the time of the extreme event

Figure 2: Visualization of an extreme event of $\omega_{z}$ at $R a=2.5 \times 10^{5}$ from a time evolution serie at the position of the extreme event $\left(x_{e}, y_{e}\right)$ and a snapshot of $\omega_{z}$. $\sigma$ indicates the standard deviation and $T_{f f}$ the free fall time of the flow. Arrows in plot (b) show the in-plane velocity.

Probability Density Functions (PDFs) of $\omega_{z}$ from stereo PIV experiments and from DNS data are shown respectively in figure 1(a) and 1(b) for all Rayleigh numbers studied. We can observe that for both kind of data the tails of the PDF becomes wider while increasing the Rayleigh number, which may be connected to intermittency. This crossover from Gaussian to intermittent statistic was recently studied in Valori and Schumacher (2021) from DNS. Figure2(a) shows the temporal evolution of $\omega_{z}$ at the position of its largest (extreme) value at $R a=2.5 \times 10^{5}$, while figure 2(b) shows the spatial distribution of $\omega_{z}$ at the time of its extreme event in the experiments.

The experimental results are able to reproduce well the statistics of DNS data of the same flow, and allow the study of extreme events of $\omega_{z}$.

\section{Acknowledgements}

The work is supported by the Priority Programme DFG-SPP 1881 on Turbulent Superstructures of the Deutsche Forschungsgemeinschaft, and the ConVext Marie Skłodowska-Curie Project Number 101024531 funded by the European Union.

\section{References}

Cierpka C, Kästner C, Resagk C, and Schumacher J (2019) On the challenges for reliable measurements of convection in large aspect ratio Rayleigh-Bénard cells in air and sulfur-hexafluoride. Experimental Thermal and Fluid Science 109:109841

Kästner C, Resagk C, Westphalen J, Junghähnel M, Cierpka C, and Schumacher J (2018) Assessment of horizontal velocity fields in square thermal convection cells with large aspect ratio. Experiments in Fluids 59:171

Valori V (2018) Rayleigh-Bénard convection of a supercritical fluid. doctoral thesis. Delft University of Technology

Valori V, Elsinga GE, Rohde M, Westerweel J, and van der Hagen THJJ (2019) Particle image velocimetry measurements of a thermally convective supercritical fluid. Experiments in Fluids 60:143

Valori V and Schumacher J (2021) Connecting boundary layer dynamics with extreme bulk dissipation events in Rayleigh-Bénard flow. Europhysics Letters, in press 